\title{
Global Convexity Graph of a Graph
}

\section{K. Karuppasamy, S. Arumugam}

\begin{abstract}
Let $G=(V, E)$ be a graph. A function $g: V \rightarrow[0,1]$ is called a global dominating function (GDF) of $G$, if for every $v \in V, g(N[v])=\sum_{u \in N[v]} g(u) \geq 1$ and $g(\overline{N(v)})=\sum_{u \notin N(v)} g(u) \geq 1$. A GDF $g$ of a Graph $G$ is minimal (MGDF) if for all functions $f: V \rightarrow[0,1]$ such that $f \leq g$ and $f(v) \neq g(v)$ for at least one $v \in V, f$ is not a GDF. In this paper, we introduce the concept of global convexity graph and determine the global convexity graphs for some standard graphs.
\end{abstract}

Keywords: convexity graph, global convexity graph, global dominating function, global domination.

\section{INTRODUCTION}

Let $G=(V, E)$ be finite, undirected simple graph with $p$ vertices and $q$ edges. For basic terminology in graphs we refer to Chartrand and Lesniak [2].

A comprehensive treatment of the fundamentals of domination in graphs is given in the book by Haynes et al. [4]. Surveys several advanced topics on domination is given in the book edited by Haynes et al. [5].

Sampathkumar [7] introduced the concept of global domination.

A dominating set $D$ of $G=(V, E)$ is a global dominating set of $G$ if $D$ is also a dominating set of the complement $\bar{G}$ of $G$. The minimum cardinality of a global dominating set of $G$ is called the global domination number of $G$ and is denoted by $\gamma_{g}(G)$ or simply $\gamma_{g}$.

In [1] we have introduced the concept of fractional global domination.

Definition 1.1:[1] A function $g: V \rightarrow[0,1]$ is called a global dominating function (GDF) of a graph $G=(V, E)$ if for every $v \in V, g(N[v])=\sum_{u \in N[v]} g(u) \geq 1$ and $g(\overline{N(v)})=\sum_{u \notin N(v)} g(u) \geq 1$.

A GDF $g$ of a graph $G$ is called minimal (MGDF) if for all functions $f: V \rightarrow[0,1]$ such that $f \leq g$ and $f(v) \neq g(v)$ for at least one $v \in V, f$ is not a GDF. The fractional global domination number $\gamma_{f g}(G)$ and the upper fractional global domination number $\Gamma_{f g}(G)$ are defined as follows:

$\gamma_{f g}(G)=\min \{|g|: g$ is an MGDFof $G\}$ and

Revised Manuscript Received on December 15, 2019.

* Correspondence Author Research and Education, Krishnankoil, Virudhunagar District, Tamilnadu, India. Email: karuppasamyk@gmail.com

Dr. S. Arumugam, Mathematics, Kalasalingam Academy of Research and Education, Krishnankoil, Virudhunagar District, Tamilnadu, India. Email: s.arumugam.klu@gmail.com
Dr. K. Karuppasamy*, Mathematics, Kalasalingam Academy of

$$
\Gamma_{f g}(G)=\max \{g \mid: g \text { is a MGDFof } G\} \text {. }
$$

Definition 1.2: [1] Let $\mathrm{f}$ be a GDF of a graph G. The positive set $P_{f}$ of $f$ and the boundary set $B_{f}$ of $f$ are defined as $P_{f}=\{v \in V: f(v)>0\} \quad$ and $\quad B_{f}=N_{f} \cup \overline{N_{f}} \quad$ where $N_{f}=\left\{v \in V: \sum_{w \in N[v]} f(w)=1\right\}, \overline{N_{f}}=\left\{v \in V: \sum_{w \notin N(v)} f(w)=1\right\}$.

Definition 1.3: Let $f$ be a GDF of a graph with positive set $P_{f}$ and boundary set $B_{f}$. We say that $B_{f}$ globally dominates $P_{f}$ if for every vertex $v \in P_{f}-B_{f}$, there exists a vertex $u \in N_{f}$ such that $u$ is adjacent to $v$ or there exists a vertex $u \in \overline{N_{f}}$ such that $u$ is not adjacent to $v$ and we write $B_{f} \underset{g}{\longrightarrow} P_{f}$.

Theorem 1.4: [1] A GDF $f$ of a graph $G$ is an MGDF if and only if $B_{f} \underset{g}{\longrightarrow} P_{f}$.

Definition 1.5: Let $f$ and $g$ be GDFs of $G$ and let $0<\lambda<1$. Then $h_{\lambda}=\lambda f+(1-\lambda) g$ is called a convex combination of $f$ and $g$.

Theorem 1.6: [1] Let $f$ and $g$ be two minimal GDFs of $G$ and let $0<\lambda<1$. Then $h_{\lambda}=\lambda f+(1-\lambda) g$ is a minimal GDF of $\mathrm{G}$ if and only if $\left(N_{f} \cap N_{g}\right) \cup\left(\overline{N_{f}} \cap \overline{N_{g}}\right) \underset{g}{\longrightarrow} P_{f} \cup P_{g}$.

The above theorem shows that if $f$ and $g$ are MGDFs of $G$ then either all convex combinations of $f$ and $g$ are MGDFs or no convex combination of $f$ and $g$ is an MGDF. Hence analogous to the concept of convexity graph with respect to MDFs introduced by Cockayne et al. [3], we introduce the concept of global convexity graph with respect to MGDFs and initiate a study of the same. We determine the global convexity graphs of some standard graphs.

\section{GLOBAL CONVEXITY GRAPH}

Let $\Omega$ denote the set of all MGDFs of a graph $G$. We define a relation $\rho$ on $\boldsymbol{\Omega}$ as follows. For $f, g \in \boldsymbol{\Omega}, f \boldsymbol{\rho} g$ if and only if $N_{f}=N_{g}, \overline{N_{f}}=\overline{N_{g}}$ and $P_{f}=P_{g}$. Clearly $\rho$ is an equivalence relation on $\Omega$ and $\Omega$ is partitioned into a finite number of equivalence classes.

Definition 2.1: Let $X=\left\{X_{1}, X_{2}, \cdots, X_{t}\right\}$ be the set of all equivalence classes of $\Omega$ with respect to the equivalence relation $\rho$. We define the global convexity graph (GCG) of $G$, denoted by $C_{g}(G)$, by $V\left(C_{g}(G)\right)=X=\left\{X_{1}, X_{2}, \cdots, X_{t}\right\}$ and $X_{i}, X_{j}$ are adjacent if and only if there exist $f \in X_{i}$ and $g \in X_{j}$ such that any convex combination of $f$ and $g$ is an MGDF of $G$. 
We proceed to determine the global convexity graphs of some standard graphs. The following observation is useful in this regard.

Observation 2.2: If there exists a dominating set $S$ of $G$ such that $N_{f}$ contains $S$ for all MGDFs $f$ of $G$ or if there exists a dominating set $T$ of $\bar{G}$ such that $\overline{N_{f}}$ contains $\mathrm{T}$ for all MGDFs $f$ of $G$, then it follows from Theorem 1.6 that $C_{g}(G)$ is complete.

Observation 2.3: For any complete graph $K_{n}$, we have $C_{g}\left(K_{n}\right) \cong K_{1}$. In fact if $g$ is any MGDF of $K_{n}$, then $g(v)=1$ for all $v \in V\left(K_{n}\right)$.

Theorem 2.4: For the complete bipartite graph $G=K_{r, s}$, we have $C_{g}(G) \cong K_{\left(2^{r}-1\right)\left(2^{s}-1\right)}$.

Proof: Let $(X, Y)$ be the bipartition of $\mathrm{G}$ with $|X|=r$ and $|Y|=s$. Let $S$ and $T$ be nonempty subjects of $X$ and $Y$ respectively.

$$
\text { Define } \quad g: V(G) \rightarrow[0,1] \quad \text { by } \quad g(u)=\frac{1}{|S|} \text { if } u \in S
$$

$g(u)=\frac{1}{|T|}$ if $u \in T ; g(u)=0$ otherwise

Then $g$ is an MGDF of $G$ with $P_{g}=S \cup T, N_{g}=V(G)-(S \cup T)$ and $\overline{N_{g}}=X \cup Y=V(G)$. Thus each pair $(S, T)$ where $S$ is a nonempty subset of $X$ and $T$ is a nonempty subset of $Y$ gives an MGDF of $\mathrm{G}$ and if $\left(S_{1}, T_{1}\right) \neq\left(S_{2}, T_{2}\right)$, then the corresponding MGDFs determine two distinct equivalence classes. Thus we have $\left(2^{r}-1\right)\left(2^{s}-1\right)$ equivalence classes of MGDFs.

We now claim that these are the only equivalence classes of MGDFs for $G$. Let $f$ be any MGDF of $G$ with $P_{f}=S^{\prime} \cup T^{\prime}$ where $S^{\prime} \subseteq X$ and $T^{\prime} \subseteq Y$. Clearly if $u \in X$, then $\sum_{v \notin N(u)} f(v)=\sum_{v \in S^{\prime}} f(v) \geq 1$. Similarly, $\quad \sum_{v \in T^{\prime}} f(v) \geq 1$. It follows from the minimality that $\sum_{v \in S^{\prime}} f(v)=1$ and $\sum_{v \in T^{\prime}} f(v)=1$. Hence the number of equivalence classes of MGDFs of $G$ is $\left(2^{r}-1\right)\left(2^{s}-1\right)$. Also $\overline{N_{f}}=V$ for all MGDFs and it follows from Observation 2.2 that $C_{g}(G)$ is complete. Thus $C_{g}(G) \cong K_{\left(2^{r}-1\right)\left(2^{s}-1\right)}$.

Corollary 2.5: For the star $K_{1, n}$, we have $C_{g}\left(K_{1, n}\right) \cong K_{2^{n}-1}$.

Theorem 2.6: For the wheel $W_{5}=K_{1}+C_{4}$, we have $C_{g}\left(W_{5}\right) \cong K_{9}$.

Proof: Let $V\left(W_{5}\right)=\left\{v_{0}, v_{1}, v_{2}, v_{3}, v_{4}\right\} \quad$ and $E\left(W_{5}\right)=$ $\left\{v_{0} v_{i}: 1 \leq i \leq 4\right\} \cup\left\{v_{1} v_{2}, v_{2} v_{3}, v_{3} v_{4}, v_{4} v_{1}\right\}$. Let $g$ be any MGDF of $W_{5}$. Since $v_{0}$ is an isolated vertex in $\overline{W_{5}}, g\left(v_{0}\right)=1$. Also $\sum_{u \notin N\left(v_{1}\right)} g(u)=g\left(v_{1}\right)+g\left(v_{3}\right) \geq 1$ and $\sum_{u \notin N\left(v_{2}\right)} g(u)=g\left(v_{2}\right)+g\left(v_{4}\right) \geq 1$. It follows from the minimality of $g$ that $g\left(v_{1}\right)+g\left(v_{3}\right)=1$ and $g\left(v_{2}\right)+g\left(v_{4}\right)=1$.

Now, let $g\left(v_{1}\right)=\lambda_{1}$ and $g\left(v_{2}\right)=\lambda_{2}$ so that $g\left(v_{3}\right)=1-\lambda_{1}$ and $g\left(v_{4}\right)=1-\lambda_{2}$, where $0 \leq \lambda_{1}, \lambda_{2} \leq 1$. Hence there are nine equivalence classes of MGDFs of $W_{5}$. Since $g\left(v_{1}\right)+g\left(v_{3}\right)=g\left(v_{2}\right)+g\left(v_{4}\right)=g\left(v_{0}\right)=1$ it follows that $\overline{N_{g}}=V$ for all MGDF $g$. Hence it follows from Observation 2.2 that $C_{g}\left(W_{5}\right)$ is complete. Thus $C_{g}\left(W_{5}\right) \cong K_{9}$.

Theorem 2.7: For any graph $G$ on $n$ vertices with $\Delta(G)<n-1$, we have $C_{g}\left(G \circ K_{1}\right) \cong K_{3^{n}}$.

Proof: Let $V(G)=\left\{v_{1}, v_{2}, \cdots, v_{n}\right\}$ and let $u_{1}, u_{2}, \cdots, u_{n}$ be the pendent vertices of $G \circ K_{1}$ adjacent to $v_{1}, v_{2}, \cdots, v_{n}$ respectively. Define $g: V\left(G \circ K_{1}\right) \rightarrow[0,1]$ by $g\left(v_{i}\right)=\lambda_{i}$ and $g\left(u_{i}\right)=1-\lambda_{i}$ where $0 \leq \lambda_{i} \leq 1$. We claim that $g$ is an MGDF of $G \circ K_{1}$. Obviously, $\sum_{w \in N\left[u_{i}\right]} g(w)=1$ and $\sum_{w \in N\left[v_{i}\right]} g(w) \geq 1$ for all $i=1,2, \cdots, n$. Also $\sum_{w \notin N\left(u_{i}\right)} g(w) \geq 1$. Since $\operatorname{deg}\left(v_{i}\right) \leq \Delta(G)<n-1$, there exists a vertex $v_{k}$ such that $v_{k}$ is not adjacent to $v_{i}$ and hence $\sum_{w \notin N\left(v_{i}\right)} g(w) \geq g\left(v_{i}\right)+g\left(v_{k}\right)+g\left(u_{k}\right) \geq 1$. Hence $g$ is a GDF of $G \circ K_{1}$. Since $g\left(u_{i}\right)+g\left(v_{i}\right)=1$ for all $i$, it follows that $g$ is an MGDF of $G \circ K_{1}$. If $\lambda_{i}=0$, then $u_{i} \in P_{f}$ and $v_{i} \notin P_{f}$. If $\lambda_{i}=1$, then $v_{i} \in P_{f}$ and $u_{i} \notin P_{f}$. If $0<\lambda_{i}<1$, then $u_{i}, v_{i} \in P_{f}$. Thus we have $3^{n}$ MGDFs and all of them have distinct positive sets. Thus we have $3^{n}$ equivalence classes of MGDFs of $G \circ K_{1}$. We now claim that these are the only equivalence classes of MGDFs for $G \circ K_{1}$.

Let $f$ be any MGDF of $G \circ K_{1}$. Then $\sum_{w \in N\left[u_{i}\right]} f(w)=f\left(u_{i}\right)+f\left(v_{i}\right) \geq 1$. It follows from the minimality of $f$ that $f\left(u_{i}\right)+f\left(v_{i}\right)=1$ for all $i$. Hence $\left|V\left(C_{g}\left(G \circ K_{1}\right)\right)\right|=3^{n}$. Also for all MGDFs $g$ of $G \circ K_{1}, N_{g}$ contains the dominating set $\left\{u_{1}, u_{2}, \cdots, u_{n}\right\}$ of $G \circ K_{1}$. Hence it follows from Observation 2.2 that the convexity graph of $G \circ K_{1}$ is complete. Thus $C_{g}\left(G \circ K_{1}\right) \cong K_{3^{n}}$.

It follows from Corollary 2.5 that the convexity graph of the path $P_{3}$ is isomorphic to $K_{3}$. We now proceed to find the convexity graphs of the paths $P_{4}$ and $P_{5}$.

Theorem 2.8: For the path $P_{4}$, we have $C_{g}\left(P_{4}\right) \cong K_{3}$.

Proof: Let $P_{4}=\left(v_{1}, v_{2}, v_{3}, v_{4}\right)$ and let $g=\left(\lambda_{1}, \lambda_{2}, \lambda_{3}, \lambda_{4}\right)$ be any MGDF of $P_{4}$ where $g\left(v_{i}\right)=\lambda_{i}, 0 \leq \lambda_{i} \leq 1$. Then $\lambda_{1}+\lambda_{2}=1, \lambda_{3}+\lambda_{4}=1, \lambda_{1}+\lambda_{3} \geq 1$ and $\lambda_{2}+\lambda_{4} \geq 1$. Hence it follows that $\lambda_{2}=\lambda_{3}=\lambda$, so that $g=(1-\lambda, \lambda, \lambda, 1-\lambda)$ where $0 \leq \lambda \leq 1$. Hence there exist exactly three equivalence classes of MGDFs of $P_{4}$, corresponding to $\lambda=1, \lambda=0$ or $0<\lambda<1$ and for each of these MGDFs, $N_{g}$ contains the dominating set $\left\{v_{1}, v_{4}\right\}$ of $P_{4}$. Hence it follows from observation 2.2 that the global convexity graph of $P_{4}$ is complete. Hence $C_{g}\left(P_{4}\right) \cong K_{3}$.

Theorem 2.9: For the path $P_{5}$, we have $C_{g}\left(P_{5}\right) \cong 2 K_{4}+K_{3}$.

Proof: Let $P_{5}=\left(v_{1}, v_{2}, v_{3}, v_{4}, v_{5}\right)$ and $g=\left(\lambda_{1}, \lambda_{2}, \lambda_{3}, \lambda_{4}, \lambda_{5}\right)$ be any MGDF of $P_{5}$ where $g\left(v_{i}\right)=\lambda_{i}, 0 \leq \lambda_{i} \leq 1$. Clearly at least one of the vertices $v_{1}$ or $v_{2}$ and at least one of the vertices $v_{4}$ or $v_{5}$ are in $P_{g}$. Let $S=\left\{v_{1}, v_{2}, v_{4}, v_{5}\right\}$ 
If $P_{g} \cap S=\left\{v_{1}, v_{5}\right\}$, then $\lambda_{2}=\lambda_{4}=0$ and hence we have exactly one equivalence class, namely, $g_{1}=(1,0,1,0,1)$ with $P_{g_{1}}=\left\{v_{1}, v_{3}, v_{5}\right\}, N_{g_{1}}=\left\{v_{1}, v_{3}, v_{5}\right\}$ and $\overline{N_{g_{1}}}=\left\{v_{2}, v_{4}\right\}$.

If $P_{g} \cap S=\left\{v_{1}, v_{4}\right\}$, then $\lambda_{5}=\lambda_{2}=0$ and hence we have exactly one equivalence class, namely, $g_{2}=(1,0,0,1,0)$ with $P_{g_{2}}=\left\{v_{1}, v_{4}\right\}, N_{g_{2}}=V$ and $\overline{N_{g_{2}}}=\left\{v_{2}, v_{3}, v_{5}\right\}$.

Similarly, if $P_{g} \cap S=\left\{v_{2}, v_{5}\right\}$, then have exactly one equivalence class, namely, $g_{3}=(0,1,0,0,1) \quad$ with $P_{g_{3}}=\left\{v_{2}, v_{5}\right\}, N_{g_{3}}=V$ and $\overline{N_{g_{3}}}=\left\{v_{1}, v_{3}, v_{4}\right\}$.

Now suppose $P_{g} \cap S=\left\{v_{2}, v_{4}\right\}$. Then $\lambda_{1}=\lambda_{5}=0$. Since $\sum_{u \notin N\left(v_{3}\right)} g(u)=g\left(v_{3}\right)$, it follows $g\left(v_{3}\right)=1$ and we have exactly one equivalence class, namely, $g_{4}=(0,1,1,1,0) \quad$ with $P_{g_{4}}=\left\{v_{2}, v_{3}, v_{4}\right\}, N_{g_{4}}=\left\{v_{1}, v_{5}\right\}$ and $\overline{N_{g_{4}}}=\left\{v_{3}\right\}$.

If $P_{g} \cap S=\left\{v_{1}, v_{2}, v_{5}\right\} g\left(v_{4}\right)=0$ and hence $g\left(v_{5}\right)=1$. Now let $g\left(v_{1}\right)=\lambda$ where $0<\lambda<1$. Then $g\left(v_{2}\right)=1-\lambda$. Now, $\sum_{u \in N\left[v_{3}\right]} g(u) \geq 1$ gives $g\left(v_{3}\right)=\lambda_{3}>\lambda$ and since $g$ is an MGDF, it follows $\lambda_{3}=\lambda$. Hence have exactly one equivalence class, namely, $\quad g_{5}=(\boldsymbol{\lambda}, 1-\lambda, \lambda, 0,1) \quad$ where $\quad 0<\lambda<1 \quad$ with $P_{g_{5}}=V-\left\{v_{4}\right\}, N_{g_{5}}=\left\{v_{1}, v_{3}, v_{5}\right\}$ and $\overline{N_{g_{5}}}=\left\{v_{4}\right\}$.

Similarly, if $P_{g} \cap S=\left\{v_{1}, v_{2}, v_{4}\right\}$, then we have exactly one equivalence class, namely, $g_{6}=(1-\lambda, \lambda, \lambda, 1,0)$ where $0<\lambda<1$ with $P_{g_{6}}=V-\left\{v_{5}\right\}, N_{g_{6}}=\left\{v_{1}, v_{5}\right\}$ and $\overline{N_{g_{6}}}=\left\{v_{3}\right\}$.

If $P_{g} \cap S=\left\{v_{1}, v_{4}, v_{5}\right\}$, then we get exactly one equivalence class, namely, $g_{7}=(1,0, \lambda, 1-\lambda, \lambda)$ where $0<\lambda<1$ with $P_{g_{7}}=V-\left\{v_{2}\right\}, N_{g_{7}}=\left\{v_{1}, v_{3}, v_{5}\right\}$ and $\overline{N_{g_{7}}}=\left\{v_{2}\right\}$.

If $P_{g} \cap S=\left\{v_{2}, v_{4}, v_{5}\right\}$, then we get exactly one equivalence class, namely, $g_{8}=(0,1, \lambda, \lambda, 1-\lambda)$ where $0<\lambda<1$ with $P_{g_{8}}=V-\left\{v_{1}\right\}, N_{g_{8}}=\left\{v_{1}, v_{5}\right\}$ and $\overline{N_{g_{8}}}=\left\{v_{3}\right\}$.

Now let $P_{g} \cap S=\left\{v_{1}, v_{2}, v_{4}, v_{5}\right\}=S$. Then $0<\lambda_{1}<1$, $\lambda_{2}=1-\lambda_{1}, 0<\lambda_{5}<1, \lambda_{4}=1-\lambda_{5}$. we claim that in this case, there exist three equivalence classes of MGDFs.

If $\lambda_{3}=0$, then $\sum_{u \notin N\left(v_{3}\right)} g(u) \geq 1$ implies that $\lambda_{1}+\lambda_{5} \geq 1$, so that $\lambda_{5} \geq 1-\lambda_{1}$. Since $g$ is an MGDF, $\lambda_{5}=1-\lambda_{1}$. Hence we get an equivalence class $g_{9}=\left(\lambda_{1}, 1-\lambda_{1}, 0, \lambda_{1}, 1-\lambda_{1}\right)$ where $0<\lambda_{1}<1$ with $P_{g_{9}}=V-\left\{v_{3}\right\}, N_{g_{9}}=V$ and $\overline{N_{g_{9}}}=\left\{v_{3}\right\}$.

Now suppose $\lambda_{3}>0$. Then $\sum_{u \notin N\left(v_{3}\right)} g(u) \geq 1$ gives $\lambda_{3} \geq 1-\lambda_{1}-\lambda_{5}$. Also $\sum_{u \in N\left[v_{3}\right]} g(u) \geq 1$ gives $\lambda_{3} \geq 1-\lambda_{2}-\lambda_{4}=$ $\lambda_{1}+\lambda_{5}-1$. Since $g$ is an MGDF of $G$ it follows $\lambda_{3}=\max \left\{1-\lambda_{1}-\lambda_{5}, \lambda_{1}+\lambda_{5}-1\right\}$. Also since $\lambda_{3}>0$, it follows that $\lambda_{1}+\lambda_{5} \neq 1$. Hence we have two equivalence classes of MGDFs according as $\lambda_{1}+\lambda_{5}<1$ or $\lambda_{1}+\lambda_{5}>1$ and they are given by $g_{10}=\left(\lambda_{1}, 1-\lambda_{1}, 1-\lambda_{1}-\lambda_{5}, 1-\lambda_{5}, \lambda_{5}\right) \quad$ where $0<\lambda_{1}, \lambda_{5}<1$ and $0<\lambda_{1}+\lambda_{5}<1$ with $P_{g_{10}}=V, N_{g_{10}}=\left\{v_{1}, v_{5}\right\}$ and $\overline{N_{g_{10}}}=\left\{v_{3}\right\}$ and $g_{11}=\left(\lambda_{1}, 1-\lambda_{1}, \lambda_{1}+\lambda_{5}-1,1-\lambda_{5}, \lambda_{5}\right)$ where

$0<\lambda_{1}, \lambda_{5}<1$ and $\lambda_{1}+\lambda_{5}>1$ with $P_{g_{11}}=V, \quad N_{g_{11}}=\left\{v_{1}, v_{3}, v_{5}\right\}$ and $\overline{N_{g_{11}}}=\phi$.

Thus there are exactly 11 equivalence classes of MGDFs for $P_{5}$. Let $A=\left\{g_{4}, g_{6}, g_{8}, g_{10}\right\}, \quad B=\left\{g_{1}, g_{5}, g_{7}, g_{11}\right\}$ and $C=\left\{g_{2}, g_{3}, g_{9}\right\}$.

By using Theorem 1.6, it can be easily verified that $\langle A\rangle=\langle B\rangle \cong K_{4},\langle C\rangle=K_{3}$ and $\langle A \cup B\rangle=2 K_{4}$. Also every element of $A \cup B$ is adjacent to every element of $C$. Hence $\langle A \cup B \cup C\rangle \cong\left(2 K_{4}+K_{3}\right)$ and thus $C_{g}\left(P_{5}\right) \cong\left(2 K_{4}+K_{3}\right)$.

\section{CONCLUSION}

In this paper we have introduced the concept of global convexity graphs and determined the global convexity graphs of some standard graphs. An interesting problem is to investigate properties of the global convexity graphs of specific families of graphs such as trees and cycles. Also the problem of determining the global convexity graphs of arbitrary paths and cycles remains open.

\section{REFERENCES}

1. S. Arumugam, K. Karuppasamy, I. S. Hamid, "Fractional Global Domination in Graphs", Discuss. Math. Graph Theory, 30(2010), 33 44.

2. G. Chartrand and L. Lesniak, Graphs and Digraphs, Fourth Ed., Chapman and Hall, CRC, 2005.

3. E. J. Cockayne, C. M. Mynhardt and B. Yu, "Universal minimal total dominating functions in graphs", Networks, 24(1994), 83 - 90.

4. T. W. Haynes, S. T. Hedetniemi and P. J. Slater, Fundamentals of Domination in Graphs, Marcel Dekker, Inc (1998).

5. T. W. Haynes, S. T. Hedetniemi and P. J. Slater, Domination in Graphs: Advanced Topics, Marcel Dekker, Inc (1998).

6. S. M. Hedetniemi, S. T. Hedetniemi and T. V. Wimer, "Linear time resource allocation algorithms for trees", Technical Report URI - 014 Department of Mathematics, Clemson University (1987).

7. E. Sampathkumar, "The global domination number of a graph", J. Math Phys. Sci. 23(1989), 377 - 385

\section{AUTHORS PROFILE}

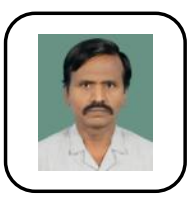

Dr. K. Karuppasamy, Professor, Department of Mathematics completed his Master's degree in Mathematics, Computer Applications and Education and Doctoral degree in Mathematics. Has wide experience in teaching and administration. Already served as the Head of the Department of Mathematics and Deputy Controller of Examinations. Acted as a Quiz master in Mathematics. Active member of ISTE. Participated many workshops and conferences thorough out India.

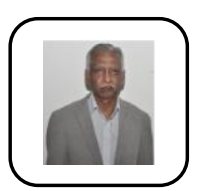

Dr. S. Arumugam, Director, n-CARDMATH, Kalsalingam Academy of Research and Education, Adjunct Professor at Ball State University, Muncie, USA and Adjunct Professor at Amrita Vishwa Vidyapeetham, Coimbatore. Has completed his Master and Doctoral degree in Mathematics having more than 50 years of Teaching, Research and Administrative experience, published more than 250 research papers, 20 text books, Edited 14 proceedings of conferences and workshops, organized 28 conferences and workshops, delivered more than 370 invited talks in various conferences and workshop inside and outside India, guided $44 \mathrm{Ph} . \mathrm{D}$. scholars as a research supervisor, received many awards and completed many funded projects, Life member of Indian Mathematical Society, Ramanujan Mathematical Society, Calcutta Mathematical Society, ISTE, ADMA and member of American Mathematical Society, Mathematical Association of America and Institute of Combinatorics and its Applications. 